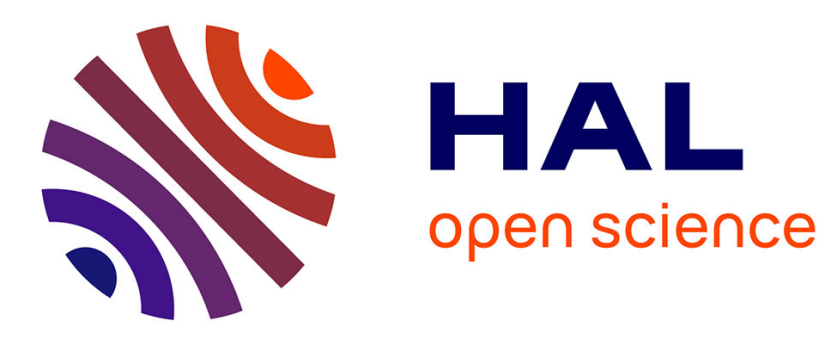

\title{
Efforts en faveur de l'Alisier torminal (et du Cormier) en Allemagne
}

\author{
Wedig Kausch-Blecken von Schmeling
}

\section{To cite this version:}

Wedig Kausch-Blecken von Schmeling. Efforts en faveur de l'Alisier torminal (et du Cormier) en Allemagne. Revue forestière française, 1993, 45 (3), pp.357-363. 10.4267/2042/26432 . hal-03444017

\section{HAL Id: hal-03444017 \\ https://hal.science/hal-03444017}

Submitted on 23 Nov 2021

HAL is a multi-disciplinary open access archive for the deposit and dissemination of scientific research documents, whether they are published or not. The documents may come from teaching and research institutions in France or abroad, or from public or private research centers.
L'archive ouverte pluridisciplinaire HAL, est destinée au dépôt et à la diffusion de documents scientifiques de niveau recherche, publiés ou non, émanant des établissements d'enseignement et de recherche français ou étrangers, des laboratoires publics ou privés. 


\section{L'ALISIER DANS \\ LES PAYS VOISINS}

\section{EFFORTS EN FAVEUR DE L'ALISIER TORMINAL (ET DU CORMIER) EN ALLEMAGNE}

\section{W. KAUSCH-BLECKEN VON SCHMELING}

\section{CONNAISSANCE DE LA RÉPARTITION DE L'ALISIER TORMINAL}

Au $X I X^{e}$ siècle, le passage quasi général au régime de la futaie régulière sonnait le glas et signifiait l'expulsion des Alisiers torminaux et des Cormiers des forêts allemandes, au vu et au su des forestiers, qui en parlaient franchement dans la littérature forestière de l'époque. Forcés d'augmenter la production en volume, on était prêt à s'accommoder des effets concomitants.

Jusqu'alors et même au courant du XIX siècle, alors que des matériaux en métal commençaient à être disponibles en masse, les bois durs et résistants, comme celui de l'Alisier torminal, avaient été irremplaçables pour de nombreux usages mécaniques. Dès lors, la diminution rapide de ces bois n'avait plus rien d'inquiétant, et cela d'autant moins que les petites propriétés privées, qui tardaient à convertir les taillis-sous-futaie en futaie, en assuraient encore l'approvisionnement pendant quelque temps. Plus tard, cette fonction passait peu à peu aux taillis-sousfutaie français.

En parcourant les revues forestières allemandes, on tombera, hélas, sur tel ou tel article déplorant le triste sort de l'Alisier torminal ou sur tel appel à la relance de la régénération des essences rares, comme le Cormier, l'lf... mais ils passaient tous presque inaperçus et se perdaient sans résultat.

Depuis quelques années, les sylvicultures dites "proches de la nature " regagnent du terrain en Allemagne, amenant avec elles la mise en valeur de la richesse en essences forestières indigènes stationnellement intégrables. L'Alisier torminal commence effectivement à en profiter. D'autres impulsions très importantes sont à attribuer au recensement de la répartition actuelle de cette essence, et aussi du Cormier, par des étudiants forestiers. Après vingt ans d'expérience, on peut affirmer que, chaque fois, le nombre des tiges indiquées avant le recensement soigneux sera au moins doublé et que, chaque fois, l'enquête constituera en même temps une sensibilisation très efficace des forestiers locaux. 


\section{W. KAUSCH-BLECKEN VON SCHMELING}

C'est en particulier aux lisières des forêts et aux bords des chemins et des routes que l'Alisier torminal a réussi à se maintenir (photos 1 à 3 , ci-dessous). Les rares exemplaires présents à l'intérieur des massifs témoignent du soin protecteur d'un forestier qui a admiré leur rareté ou qui a reconnu leur valeur pour leur réserver une deuxième vie (photos 4 à 6, p. 359). Dans quelques rares cas, l'Alisier torminal atteint en Allemagne des dimensions très remarquables avec des hauteurs de $33 \mathrm{~m}$ et des diamètres à $1,30 \mathrm{~m}$ du sol de $90 \mathrm{~cm}$.

Sur certaines stations et par une éducation convenable, l'Alisier torminal se maintient avec succès dans les peuplements (photo 8, p. 359) et, grâce à son drageonnement abondant, dans les régénérations (photo 7, p. 359).

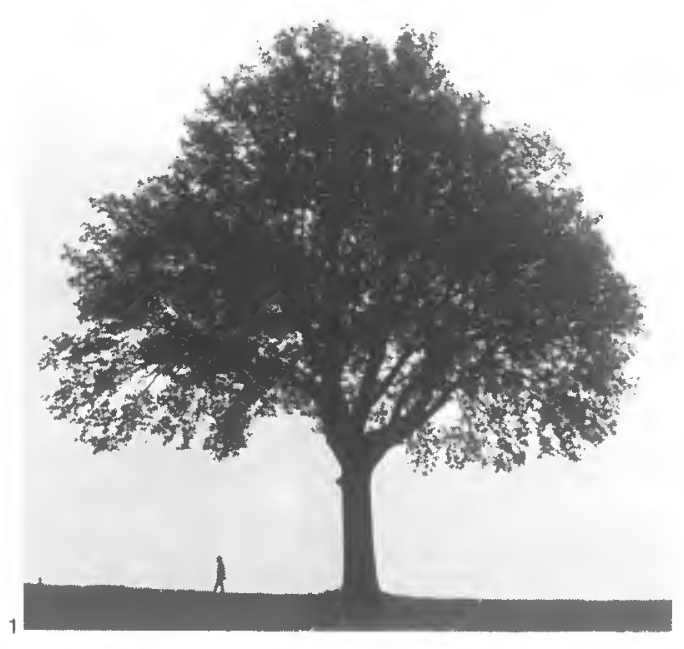

Photos KAUSCH-BLECKEN VON SCHMELING

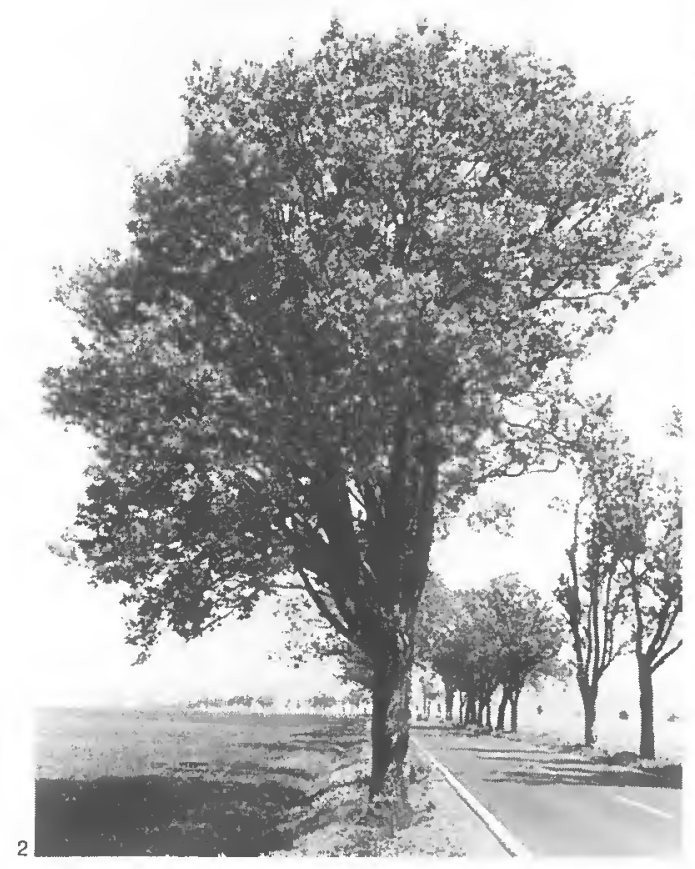

Photo 1 Alisier solitaire. Les paysagistes ont trop Iongtemps négligé l'Alisier torminal dont la parure automnale incite à l'utiliser plus fréquemment en ornement.

Photo 2 Allée d'Alisiers. Au début du $20^{\complement}$ siècle, on a créé des allées d'Alisiers torminaux près de Kassel (Hesse).

Photo 3 Alignement d'Alisiers. Alignement d'Alisiers torminaux au bord d'une route prés de Kassel (Hesse).

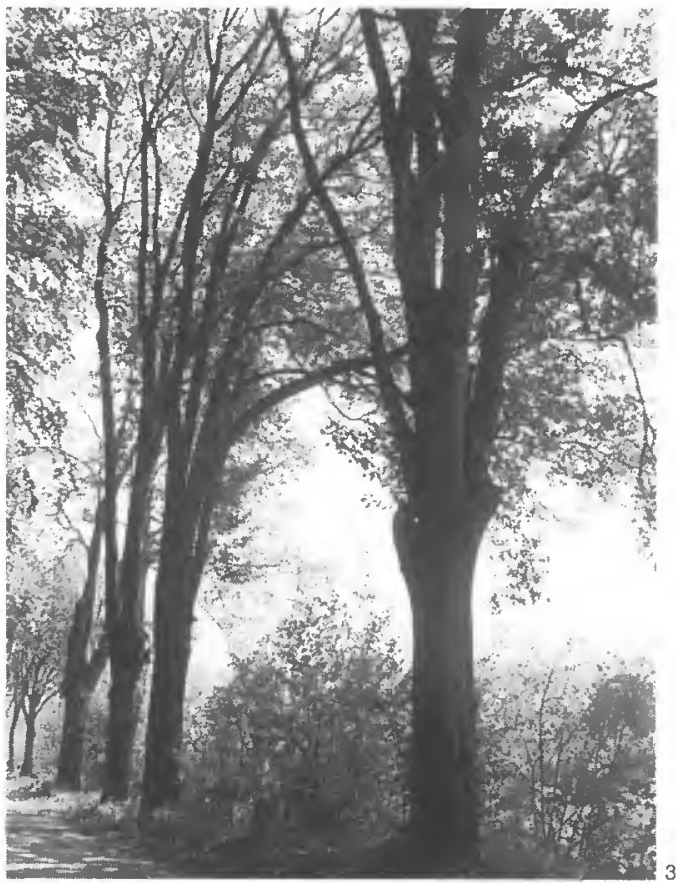


De plus grande rarete̊ parmi les feuillus allemands de qualité, le Cormier se rencontre souvent en compagnie de l'Alisier torminal (photo 5, p. 359), ce qui n'est pas du tout étonnant. Les exigences stationnelles et les aires naturelles de répartition de ces deux Sorbus se ressemblent beaucoup.

\section{MISE AU POINT DES OBJECTIFS ET DES PRATIQUES SYLVICOLES}

L'inscription de l'Alisier torminal dans les types de peuplements, fixée par les directives d'aménagement des administrations forestières des "Länder" allemands, joue un grand rôle, parce qu'ils incitent les gestionnaires à tirer un meilleur profit de cette essence. En fait, toutes ces administrations, qui jouissent encore de la présence relictuelle de l'Alisier torminal, encouragent son traitement préférentiel et sa régénèration naturelle et artificielle, surtout dans les chênaies-charmaies thermophiles et dans les hêtraies calcicoles xéroclines.

Dans l'aire naturelle de l'Alisier torminal en Allemagne du Sud, sa mise en lumière immédiate et forte serait très efficace dans de nombreuses forêts. II est à favoriser dans toutes les classes d'âges. De plus, il faut absolument s'abstenir de la préférence absolue accordée au Chêne, qui était de règle jusqu'à présent, pour sacrifier en faveur d'un Alisier torminal tel Chêne, tel Hêtre, tel Frêne, même de qualitẻ irréprochable. Si, à ce sujet, les manières d'agir ne changent pas fondamentalement, toutes les précisions d'objectifs sylvicoles ne serviront à rien.

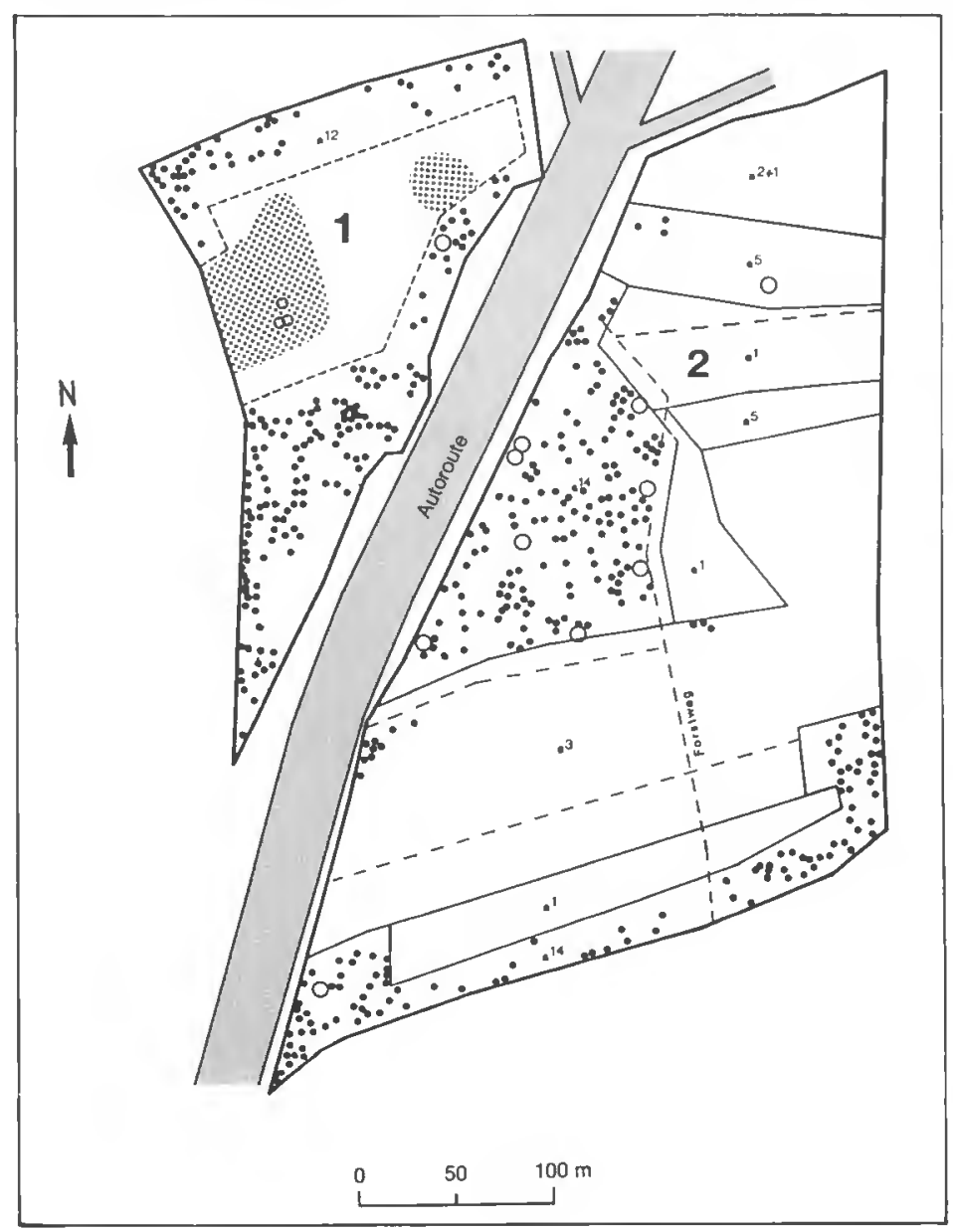

Figure 1

UN DES PEUPLEMENTS PARMI

LES PLUS RICHES EN CORMIERS

ET EN ALISIERS TORMINAUX

D'ALLEMAGNE EN FORÊT

COMMUNALE DE WEINSBERG

(BADE-WÜRTEMBERG).

L'inventaire par B. Georg met à

l'évidence, comment les Sorbus, originairement présents dans

toute la forêt, reculent du fait du traitement sylvicole et de la construction d'une autoroute. Ils ne se maintiennent ici et là qu'en sous-étage des peuplements constitués d'essences de lumière. 
Trop rares sont encore les praticiens qui savent réussir la régénération de l'Alisier torminal par drageonnement. Trop souvent, en entamant la régénération d'un peuplement, le traitement spécial de cette essence, qui est encore présente en sous-étage ou au stade de codominant, est manqué. L'Alisier torminal est malheureusement éliminé au cours des coupes de préparation à la régénération. Dans l'année suivant une telle coupe, de nombreux drageons jaillissent en général des racines traçantes des alisiers enlevés. Hélas, sans protection efficace immédiate par engrillagement, ces drageons seront complètement consommés par le gibier. Quelques années plus tard, quand le grillage est posé pour la protection des semis des essences principales, l'Alisier torminal aura déjà disparu. II faut donc insister sur l'importance de l'engrillagement systématique et précoce de l'aire de drageonnement des Alisiers torminaux abattus. C'est ainsi qu'on pérennisera cette essence tout en lui conférant une avance de développement juvénile par rapport aux autres essences.

Les effets des pratiques sylvicoles traditionnelles sont, de façon exemplaire, illustrés par la figure 1 (p. 360). Dans la forêt en question, le passage au traitement en futaie classique a détruit, pour sa plus grande part, une des plus riches stations de Sorbus en Allemagne. Ce n'est que dans les peuplements constitués par des essences de lumière, comme le Pin sylvestre, que, fort heureusement, l'Alisier torminal arrive à se maintenir et même, parfois, à rattraper l'essence principale.

\section{PRODUCTION DE PLANTS, TEST DE PROVENANCES, VERGERS A GRAINES}

En Allemagne, la production systématique et en quantités importantes de plants d'Alisier torminal n'a été abordée qu'à partir de 1970. En même temps, on lançait une expérience comparative de provenances.

Préalablement à cet essai, on a optimisé les techniques de traitement des graines. Dés lors, 100 graines donneront naissance à 40 plants viables.

Pour les expériences de provenances, on a choisi et récolté 70 arbres dans les massifs forestiers suivants: Bar-le-Duc (France), Diekirch (Luxembourg), Zbraslav (Tchécoslovaquie), Baden (Autriche), Schweinfurt, Würzburg, Sailershausen, Lutter et Göttingen (Allemagne).

232000 graines ont permis la production de 130000 plants que l'on a répartis sur trois dispositifs expérimentaux comparatifs (photos 9 à 11, p. 362). La croissance initiale des semis est beaucoup plus lente que celle des drageons. À l'âge de 15 ans, seuls les plants vigoureux ont atteint une hauteur de $5 \mathrm{~m}$ (photo 9). La proportion d'environ $25 \%$ d'arbres à fûts élancés et sans fourchaison suffit pour éduquer des peuplements de bonne qualité. Pour y arriver, il ne faut pas dépasser en plantation une densité initiale de 1000 plants/ha.

Les premiers résultats des expériences de provenances ont été publiés en 1980. Les prochaines mesures dans les trois stations qui diffèrent quant à leur dispositif et à leur conduite sylvicole sont prévues pour 1993 (DEA Stannehl). Une analyse approfondie ne sera possible que dans quelques années. Actuellement quelques arbres français rejoignent les provenances de Würzburg, de Schweinfurt et de Sailershausen dans le peloton de tête de l'expérience comparative.

Les difficultés permanentes d'approvisionnement en graines d'Alisier torminal ont conduit les États de Rhénanie-Nord-Westphalie, de Hesse et de Rhénanie-Palatinat à créer chacun un verger à graines. Les jeunes alisiers torminaux greffés et plantés à grand écartement y fructifient déjà après peu d'années. La forte demande en graines d'Alisier torminal encourage la création d'autres vergers à graines.

Une plantation expérimentale, gravement endommagée par les petits rongeurs, s'est récemment transformée en "verger à graines", du fait de l'écartement des arbres. En 1992, elle a commencé à produire des quantités importantes de graines. 


\section{FORMATION DU PERSONNEL FORESTIER, PUBLICITÉ}

C'est particulièrement dans les écoles de formation des techniciens (Fachhochschulen) que les Sorbus sont traités à fond. On encourage les futurs torestiers, d'une part à tavoriser précocement et fortement les Alisiers torminaux et les Cormiers qui nous restent encore, d'autre part à réintroduire ces essences sur les stations qui leur conviennent. La préservation de ces espéces et la réputation éminente de leurs bois sont des arguments très motivants.

Une vingtaine de DEA ont èté consacrés à l'Alisier torminal et au Cormier. Les efforts des étudiants ont ainsi produit des effets d'initiation et de sensibilisation du personnel forestier concerné, mais aussi des émulations de recherches. Ils ont tacitement influé sur les décisions sylvicoles lors des futurs martelages et, aux arbres recensés, plus nombreux que ce qu'on croyait trouver, ils ont procuré de cette manière l'espace vital qui leur était nécessaire.

En Allemagne, le Cormier a été proclamé " arbre de l'année 1993 ». Ainsi, il jouira d'actions publicitaires exceptionnelles. On estime que l'Alisier torminal, en tant que proche parent, en profitera également et regagnera l'attention des forestiers, dendrologues, botanistes, pépiniéristes, jardiniers, enseignants, journalistes...

Prof. Dr. W. KAUSCH-BLECKEN VON SCHMELING

Liegnitzer Str. 17

D-3406 BOVENDEN

Texte traduit par G.J. WILHELM 